\title{
Bayesian Multiple Change-Point Estimation of Multivariate Mean Vectors for Small Data
}

\author{
Sooyoung $\mathrm{Cheon}^{1} \cdot$ Wenxing $\mathrm{Yu}^{2}$ \\ ${ }^{1}$ Department of Informational Statistics, Korea University \\ ${ }^{2}$ Department of Economics and Statistics, Korea University \\ (Received July 31, 2012; Revised August 27, 2012; Accepted October 23, 2012)
}

\begin{abstract}
A Bayesian multiple change-point model for small data is proposed for multivariate means and is an extension of the univariate case of Cheon and $\mathrm{Yu}$ (2012). The proposed model requires data from a multivariate noncentral $t$-distribution and conjugate priors for the distributional parameters. We apply the MetropolisHastings-within-Gibbs Sampling algorithm to the proposed model to detecte multiple change-points. The performance of our proposed algorithm has been investigated on simulated and real dataset, Hanwoo fat content bivariate data.
\end{abstract}

Keywords: Small data, change-point, noncentral $t$-distribution, Metropolis-Hastings-Within-Gibbs sampling, Hanwoo fat content.

\section{1. 서론}

경제학, 금융학, 기상학, 금융학 등 여러 분야에서 변환점에 대한 관심이 증가하고 있다. 하지만, 일반 적으로 변환점의 수와 위치는 알려져 있지 않기때문에, 변환점을 찾는 문제가 통계학에서 도전해 볼만 한 분야이다. 본 연구에서는 축산학에서의 변환점분석을 위해 한우자료를 다룬다. 일반적으로 한우의 지방함량따라 한우의 맛이 달라지는데, 이러한 한우의 맛을 평가하는데 있어 지방함량에 따른 부드러 움(Tenderness)과 육즙(Juiciness)이 주로 사용된다. 한우는 이제 더 이상 우리만의 것이 아니라 세계 가 주목하고 있는 글로벌 한우가 되었다. 한우가 해외시장에서 당당하게 대접받기 위해서는 한우의 맛 을 개선시킬 필요가 있다. 따라서 한우의 맛에 중요한 역할을 하는 지방함량 비율에 대한 변환점을 찾는 것이 중요한 과제이다.

일반적으로 자료가 정규분포를 따른다는 가정하에, 하나의 변환점인 경우, Chernoff와 Zacks (1964)와 Smith (1975)가 변환점 추론을 위해 베이지안 방법을 제안하였고, Carlin 등 (1992)은 마코브 연쇄 몬 테카를로(Markov chain Monte Carlo; MCMC; Metropolis 등, 1953; Hastings, 1970) 알고리즘을 이 용하여 Smith (1975)의 방법을 확장하였다. 다중 변환점 모형인 경우에, Barry와 Hartigan (1993)은 정규분포의 곱분할 모형(product partition model)에서 모수들의 평균 변화를 통해 변환점을 찾았으며, 최근에 Kim과 Cheon (2010)과 Cheon과 Kim (2010)은 stochastic approximation Monte Carlo 알

\footnotetext{
${ }^{1}$ Corresponding author: Assistant Professor, Department of Informational Statistics, Korea University, 2511 Sejong-ro, Sejong-city 339-700, South Korea. E-mail: scheon@korea.ac.kr
} 
고리즘을 이용하여 일변량과 이변량의 정규분포에서 베이지안 다중변환점 모형을 제안하였다. 이와 같 이 지금까지 제안된 대부분의 모형은 대량자료 분석을 위해 정규 분포 자료의 베이지안 다중 변환점 모 형에 $\mathrm{MCMC}$ 를 이용하여 해결하였다. 하지만 소량자료 분석을 위한 베이지안 다중 변환점 모형에 대한 연구는 아직까지 미진하다. 일반적으로 자료가 소량일때, 정규분포를 가정한 통상적인 통계적 방법으로 는 정확한 추론을 할 수 없다. 보다 정확한 추론을 위해 소량자료에 대한 연구에 있어, 최근에 Cheon과 $\mathrm{Yu}$ (2012)가 단변량 자료의 베이지안 비중심 $t$ 분포 다중 변환점 모형을 제안하였다. 이에 대한 확장으 로 본 논문에서는 다변량 자료의 베이지안 비중심 $t$ 분포 모형을 제안하여 보다 복잡한 모형에서의 변환 점 분석을 하고자 한다.

일반적으로 소량자료는 정규분포를 따르기보다 비중심(noncentral) $t$ 분포를 따르는 경향이 크다. 본 논문에서는 베이지안 다변량 비중심 $t$ 분포 모형을 제안하고 메트로폴리스-해스팅스를 포함한 깁스 샘 플링(Metropolis-Hastings-within-Gibbs Sampling; MHWGS) 알고리즘을 이용하여 소량자료에서 변 환점 분석을 하고자 한다. 본 논문에서 다루는 실증 분석 자료는 2006 년도 10 군데 서로 다른 부위에서 잘라낸 한우의 지방함량에 따른 부드러움(Tenderness)과 육즙(Juiciness)의 이변량 자료이다.

본 논문의 2 장에서 베이지안 다변량 비중심 $t$ 분포 다중 변환점 모형 및 MHWGS를 이용한 베이지안 모형 선택에 대하여 소개하고, 3 장에서는 모의실험 자료의 분석 및 그 결과를 보여준다. 4 장에서는 한우 의 이변량 자료의 실증 분석을 하며, 5 장에서는 본 논문의 결론을 정리한다.

\section{2. 베이지안 다중 변환점 분석}

\section{1. 베이지안 다변량 비중심 $t$ 분포 다중 변환점 모형}

본 논문의 베이지안 다중변환점 분석은 Cheon과 $\mathrm{Yu}$ (2012)에서 이용한 모형과 방법의 확장을 통해 분 석한다. $d \times 1$ 확률 벡터 $\vec{X}$ 가 다변량 비중심 $t$ 분포를 따른다고 할때, 변환점이 없는 다변량 $\vec{X}$ 의 확률 분포(probability density function)는 다음과 같다.

$$
f(\vec{x} \mid p, \vec{u}, \Sigma) \propto|\Sigma|^{-\frac{1}{2}}\left[1+\frac{(\vec{x}-\vec{u})^{\prime} \Sigma^{-1}(\vec{x}-\vec{u})}{p}\right]^{-\frac{p+d}{2}} .
$$

여기서 $p$ 는 자유도, $d$ 는 차원수, $\vec{u}$ 는 $d \times 1$ 비중심 모수벡터이고 $\Sigma$ 는 $d \times d$ 분산-공분산 행렬이다.

전체영역 $S=(1,2, \ldots, n)$ 가 서로 다른 분포를 가지는 여러 부분영역으로 나누어 지고, 각각의 부분영 역들은 변환점에 의해 분할된다고 하자. 임의의 $l(\in[1, n])$ 에 대해, $T=\left(t_{1}, t_{2}, \ldots, t_{n}\right)^{\prime}$ 는 $t_{c_{1}}=t_{c_{2}}=$ $\cdots=t_{c_{l}}=1$, 그 외는 0 인 이항 벡터라고 하고, $0=c_{0}<c_{1}<c_{2}<\cdots<c_{l+1}=n$ 이라 하자. 또한, $i=1,2, \ldots, l+1$ 에 대해 $C_{i}=\left\{m: c_{i-1}<m \leq c_{i}\right\}$ 이라 하자. 만약 변환점이 $k$ 개라면 다중 변환점 모 형을 다음과 같이 정의한다.

$$
f(\vec{x})=\prod_{j=1}^{n} f\left(\vec{x}_{j}\right)=\prod_{j \in C_{1}} f_{1}\left(\vec{x}_{j}\right) \times \cdots \times \prod_{j \in C_{k+1}} f_{k+1}\left(\vec{x}_{j}\right) .
$$

여기서 $c_{i-1}<j \leq c_{i}(i=1,2, \ldots, k+1)$ 에 대해 $\vec{x}_{j} \sim f_{i}\left(\cdot \mid \phi_{i}\right)$ 이다. 이때 $f_{i}$ 는 모수 $\phi_{i}=\left(\vec{u}_{i}, \Sigma\right)$ $(\in \Phi=(\vec{u}, \Sigma))$ 에 의존한다. 각 자료들은 $c_{1}+1, c_{2}+1, \ldots, c_{k}+1$ 에서 변화하므로 전체 공간을 $k+1$ 개 의 부분공간으로 분할하는 $c_{1}, c_{2}, \ldots, c_{k}$ 를 변환점(change-points)이라고 부른다.

만약 함수 $f_{i}$ 가 $d$ 차원 다변량 비중심 $t$ 분포라고 하면, 모수 $\phi_{i}$ 는 $d$ 차원 자유도 $p_{i}$, 위치벡터 $\vec{u}_{i}$ 와 분산행렬 $\Sigma$ 로 나뉜다. 따라서 $T^{(k)}$ 를 $k$ 개의 변환점을 가지는 $T$ 라고 할때 전체 모수는 $\eta^{(k)}=$ $\left(T^{(k)}, p_{1}, \vec{u}_{1}, \ldots, p_{k+1}, \vec{u}_{k+1}, \Sigma\right)$ 로 표현된다. $\vec{\phi}=(p, \vec{u}, \Sigma)$ 라 하면, 확률 벡터 $\vec{X}$ 는 비중심 모수가 
$\vec{u}$ 이고 독립인 다변량 $t$ 분포를 따른다고 한다. $k$ 개의 변환점이 있을 때, 자유도 $p, d \times 1$ 인 벡터 $\vec{u}$ 와 $d \times d$ 인 분산공분산 행렬 $\Sigma$ 를 가지는 다변량 $\vec{X}$ 의 확률분포(probability density function)는 다음과 같 다.

$$
f(\vec{x} \mid p, \vec{u}, \Sigma)=\prod_{j \in C_{1}} f_{1}\left(\vec{x}_{j} \mid p_{1}, \vec{u}_{1}, \Sigma\right) \times \cdots \times \prod_{j \in C_{k+1}} f_{k+1}\left(\vec{x}_{j} \mid p_{k+1}, \vec{u}_{k+1}, \Sigma\right)
$$

where $\quad f_{i}\left(\vec{x}_{j} \mid p_{i}, \vec{u}_{i}, \Sigma\right) \propto|\Sigma|^{-\frac{1}{2}}\left[1+\frac{\left(\vec{x}_{j}-\vec{u}_{i}\right)^{\prime} \Sigma^{-1}\left(\vec{x}_{j}-\vec{u}_{i}\right)}{p_{i}}\right]^{-\frac{p_{i}+d}{2}}, \quad i=1,2, \ldots, k+1$.

베이지안 추론을 위해 $\vec{u}_{i}(i=1,2, \ldots, k+1)$ 의 사전분포는 균일분포를, $\Sigma$ 의 사전분포는 Inverse$\mathrm{Wishart}$ 분포 $\mathrm{IW}\left(v_{0}, \Lambda_{0}^{-1}\right)$ 를 따른다고 가정한다. 각 분포의 자유도는 $p_{i}=n_{i}-1=c_{i}-c_{i-1}-1$ 이다. 따라서, 자유도 $p_{i}$ 대신 변환점 위치 $c_{i}$ 를 이용하면 자유도 $p_{i}$ 를 구할 수 있다. 본 연구는 $c_{i}$ 의 사전확률 분포로 이산균일분포를 선택하였다.

변환점이 $k$ 개 있을때, $k$ 개의 변환점 위치 $\left(c_{1}, c_{2}, \ldots, c_{k}\right)$ 와 $k+1$ 개의 비중심 모수 벡터 $\left(\vec{u}_{1}, \vec{u}_{2}, \ldots\right.$, $\left.\vec{u}_{k+1}\right)$ 가 주어진다. $\eta^{(k)}=\left(T^{(k)}, p_{1}, \vec{u}_{1}, \ldots, p_{k+1}, \vec{u}_{k+1}, \Sigma\right)$ 의 우도함수는 다음과 같다.

$$
L\left(\eta^{(k)} \mid X\right)=\prod_{i=1}^{k+1} \prod_{j=c_{i-1}+1}^{c_{i}} f_{i}\left(\vec{x}_{j} \mid p_{i}, \vec{u}_{i}, \Sigma\right)
$$

$\eta^{(k)}$ 의 사전확률분포로 다음과 같이 가정한다.

$$
\begin{aligned}
\pi\left(\eta^{(k)}\right) \propto & \pi(\Sigma) \times \pi\left(\vec{u}_{1}, \ldots, \vec{u}_{k+1}\right) \times \pi\left(\vec{c}_{1}, \ldots, \vec{c}_{k}\right) \\
= & \left|\Lambda_{0}\right|^{\frac{v_{0}}{2}} \times|\Sigma|^{-\frac{v_{0}+d+1}{2}} \times \exp \left[-\frac{1}{2} \operatorname{tr}\left(\Lambda_{0} \Sigma^{-1}\right)\right] \\
& \times \frac{1}{(b-a)^{2(k+1)}} \times I_{(a, b)}\left(\vec{u}_{1}, \ldots, \vec{u}_{k+1}\right) \times \frac{1}{(n-5)^{k}} \times I_{[3, \ldots, n-3]}\left(c_{1}, \ldots, c_{k}\right),
\end{aligned}
$$

여기서 $I(\cdot)$ 는 지시함수이다. 따라서 $\eta^{(k)}$ 의 로그사후(log-posterior) 확률분포인 베이지안 다변량 $t$ 분 포 다중 변환점모형은 다음과 같다.

$$
\begin{aligned}
\log \left(\pi\left(\eta^{(k)} \mid X\right)\right) \propto & \log \left(L\left(\eta^{(k)} \mid X\right) \pi\left(\eta^{(k)}\right)\right) \\
= & \sum_{i=1}^{k} \sum_{j=c_{i-1}+1}^{c_{i}}\left\{-\frac{1}{2} \log |\Sigma|-\frac{p_{i}+d}{2} \log \left[1+\frac{\left(\vec{x}_{j}-\vec{u}_{i}\right)^{\prime} \Sigma^{-1}\left(\vec{x}_{j}-\vec{u}_{i}\right)}{p_{i}}\right]\right\} \\
& +\frac{v_{0}}{2} \log \left|\Lambda_{0}\right|-\frac{v_{0}+d+1}{2} \log |\Sigma|-\frac{1}{2} \operatorname{tr}\left(\Lambda_{0} \Sigma^{-1}\right)-2(k+1) \log (b-a) \\
& +\log \left(I_{(a, b)}\left(\vec{u}_{1}, \ldots, \vec{u}_{k+1}\right)\right)-k \log (n-5)+\log \left(I_{[3, \ldots, n-3]}\left(c_{1}, \ldots, c_{k}\right)\right) .
\end{aligned}
$$

본 논문에서는 $d=2$ 인 경우인 이변량 비중심 $t$ 분포에 관하여 분석하였다. 따라서, $\eta^{(k)}$ 의 로그사후확 률분포인 베이지안 이변량 $t$ 분포 다중 변환점모형은 다음과 같이 구할 수 있다.

$$
\begin{aligned}
\log \left(\pi\left(\eta^{(k)} \mid X\right)\right) \propto & \sum_{i=1}^{k} \sum_{j=c_{i-1}+1}^{c_{i}}\left\{-\frac{1}{2} \log |\Sigma|-\frac{p_{i}+2}{2} \log \left[1+\frac{\left(\vec{x}_{j}-\vec{u}_{i}\right)^{\prime} \Sigma^{-1}\left(\vec{x}_{j}-\vec{u}_{i}\right)}{p_{i}}\right]\right\} \\
& +\frac{v_{0}}{2} \log \left|\Lambda_{0}\right|-\frac{v_{0}+3}{2} \log |\Sigma|-\frac{1}{2} \operatorname{tr}\left(\Lambda_{0} \Sigma^{-1}\right)-2(k+1) \log (b-a) \\
& +\log \left(I_{(a, b)}\left(\vec{u}_{1}, \ldots, \vec{u}_{k+1}\right)\right)-k \log (n-5)+\log \left(I_{[3, \ldots, n-3]}\left(c_{1}, \ldots, c_{k}\right)\right) .
\end{aligned}
$$




\section{2. 메트로폴리스-해스팅스를 포함한 깁스 샘플링과 베이지안 모형 선택}

결합분포에서 자료를 직접 얻기 힘든 경우가 발생했을 때, 일반적으로 채택-기각(acceptance-rejection) 알고리즘과 같은 맞춤형(customized) 알고리즘을 이용하여 해결하려고 노력한다. 본 논문은 Müller (1991, 1993)가 제안한 절충(compromised) 깁스 알고리즘, 즉 메트로폴리스-해스팅스를 포함한 깁스 샘플링 (Metropolis-Hastings-within-Gibbs Sampling; MHWGS) 알고리즘을 이용한다. MHWGS 알 고리즘에서 $f_{k}\left(x_{k} \mid x_{i}, i \neq k\right)$ 로 부터 표본을 추출하는데 어려움이 있는 깁스 샘플러(Gibbs sampler; Geman과 Geman, 1984)의 샘플링 단계가 Metropolis Hastings(MH; Hastings, 1970) 단계로 대체된 다.

이변량 베이지안 모형에서, 비중심 모수는 $\left(\vec{u}_{1}, \ldots, \vec{u}_{k+1}\right)$, 변환점 위치는 $\left(c_{1}, \ldots, c_{k}\right)$, 분산 모수는 $\Sigma$ 인 $k$ 개의 변환점이 주어진 경우를 고려해 보자. MHWGS 알고리즘의 자료생성 절차는 다음과 같다. 먼저 모든 변수들을 고려한 변수 $\boldsymbol{z}=\left(z_{1}, \ldots, z_{k+1}, z_{k+2}, \ldots, z_{2 k+1}, z_{2 k+2}\right)=\left(\vec{u}_{1}, \ldots, \vec{u}_{k+1}, c_{1}, \ldots\right.$, $\left.c_{k}, \Sigma\right)$ 를 설정한다.

\section{메트로폴리스-해스팅스를 포함한 깁스 샘플링:}

$i=1,2, \ldots, 2 k+2$ 이고 현재 표본이 $t$ 번째 반복 후인 $\boldsymbol{z}=\left(z_{1}^{(t+1)}, \ldots, z_{i-1}^{(t+1)}, z_{i}^{(t)}, \ldots, z_{2 k+2}^{(t)}\right)$ 라고 하 자.

1. $q_{i}\left(z_{i} \mid z_{1}^{(t+1)}, \ldots, z_{i-1}^{(t+1)}, z_{i}^{(t)}, \ldots, z_{2 k+2}^{(t)}\right)$ 로 부터 $z_{i}^{*}$ 를 생성한다.

2. $r=\frac{\pi_{i}\left(z_{i}^{*} \mid z_{1}^{(t+1)}, \ldots, z_{i-1}^{(t+1)}, z_{i+1}^{(t)}, \ldots, z_{2 k+2}^{(t)}\right)}{\pi_{i}\left(z_{i}^{(t)} \mid z_{1}^{(t+1)}, \ldots, z_{i-1}^{(t+1)}, z_{i+1}^{(t)}, \ldots, z_{2 k+2}^{(t)}\right)} \times \frac{q_{i}\left(z_{i}^{(t)} \mid z_{1}^{(t+1)}, \ldots, z_{i-1}^{(t+1)}, z_{i}^{*}, z_{i+1}^{(t)}, \ldots, z_{2 k+2}^{(t)}\right)}{q_{i}\left(z_{i}^{*} \mid z_{1}^{(t+1)}, \ldots, z_{i-1}^{(t+1)}, z_{i}^{(t)}, z_{i+1}^{(t)}, \ldots, z_{2 k+2}^{(t)}\right)}$ 계산한다.

3. $\min (1, r)$ 의 확률을 가지고 채택이면 $z_{i}^{(t+1)}=z_{i}^{*}$ 이고, 기각이면 $z_{i}^{(t+1)}=z_{i}^{(t)}$ 이다.

여기서 $\mathrm{MH}$ 절차는 각각의 반복에서 오직 한 번만 시행한다. 만약 $\mathrm{MH}$ 절차를 각각의 반복에서 여러 번 시행했을 경우 $\pi_{i}(\cdot)$ 의 더 정확한 근사치를 얻을 수 있다. 하지만 오직 한 번의 절차만 시행해도 비 교적 좋은 결과를 얻을 수 있기 때문에 효율성을 위해 본 논문에서는 오직 한번의 $\mathrm{MH}$ 절차만 시행한다 (Chen과 Schmeiser, 1998).

본 논문에서는 MHWGS 알고리즘을 이용하여 얻어진 표본으로 부터 계산된 사후 확률과 Bayesian Information Criterion(BIC)를 동시에 고려하여 최적의 모형인 최대 사후 확률(maximum a posteriori; MAP) 모형을 선택한다 (Cheon과 $\mathrm{Yu}, 2012)$.

$$
\mathrm{BIC}=-2(\log (\text { 최대 우도값 }))+(\log (\text { 자료의 수 }))(\text { 모수의 수 }) \text {. }
$$

\section{3. 모의실험}

\section{1. 모의실험 자료}

본 연구에서 제안한 모형의 모의실험을 위해 각각 20 개씩 3 개 부분으로 나뉜 60 개의 이변량 자료들 로 이루어진 서로 다른 4 개 세트를 차례로 생성시켰다 (Figure 3.1). 즉, $\vec{x}_{1}, \ldots, \vec{x}_{20} \sim \operatorname{BVT}\left(p_{1}=\right.$ $19, \vec{u}_{1}=\left(\vec{u}_{11}, \vec{u}_{12}, \vec{u}_{13}, \vec{u}_{14}\right)=((3.53,2.71),(3.53,10.62),(4.93,3.60),(3.72,8.03)) ; \vec{x}_{21}, \ldots, \vec{x}_{40} \sim$ $\operatorname{BVT}\left(p_{2}=19, \vec{u}_{2}=\left(\vec{u}_{21}, \vec{u}_{22}, \vec{u}_{23}, \vec{u}_{24}\right)=((6.29,6.78),(6.29,6.78),(7.63,8.22),(7.52,4.80)) ; \vec{x}_{41}, \ldots\right.$, $\vec{x}_{60} \sim \operatorname{BVT}\left(p_{3}=19, \vec{u}_{3}=\left(\vec{u}_{31}, \vec{u}_{32}, \vec{u}_{33}, \vec{u}_{34}\right)=((8.54,10.62),(8.54,2.71),(9.99,6.31),(9.76,7.56))\right.$. 모의실험을 위한 통계 패키지로는 R-software(version 2.12.1)를 사용하였다. 


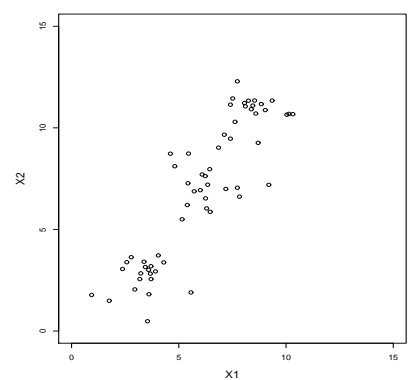

(a)

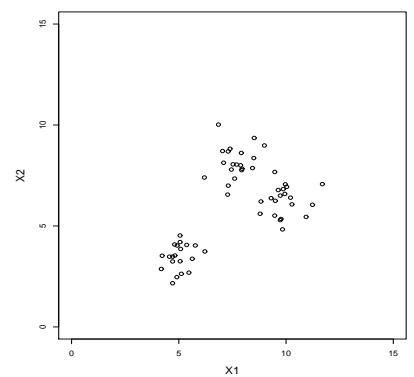

(c)

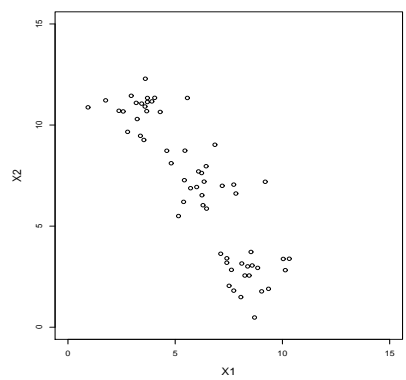

(b)

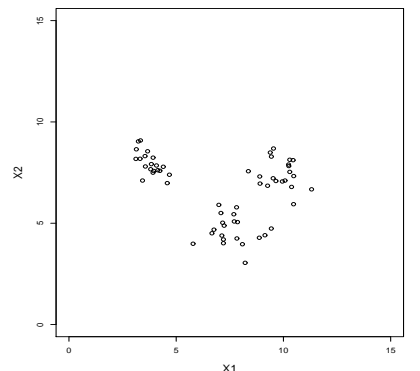

(d)

Figure 3.1. The plots of four simulated data from the bivariate $t$ distribution.

\section{2. 모의실험 결과}

본 모의실험은 변환점이 각각 1 개, 2 개, 3 개인 경우만을 고려하였다. Table 3.1 은 MHWGS 알고리즘 을 이용하여 변환점이 1 개, 2 개, 3 개인 경우와 4 개의 모의실험 자료에 대해 각각 10000 번씩 시행하여 로그사후확률이 가장 큰 5 개 값으로부터 얻은 결과이다. Table 3.1 로부터 모든 경우에 있어 변환점 2 개 에서의 로그사후확률이 다른 변환점에서의 로그사후확률보다 크고 $\mathrm{BIC}$ 값도 가장 작은 것을 볼 수 있다. 즉, 변환점 위치가 $(20,40)$ 일 때 로그사후확률값은 각각 $(-69.8243,-71.0516,-31.6007,-30.7672)$ 로 가장 크고 $\mathrm{BIC}$ 값도 각각 $(176.4977,178.9524,100.0506,98.3835)$ 으로 가장 작게 얻어졌다. 따라서, 사 후확률과 $\mathrm{BIC}$ 에 의해 $\mathrm{MAP}$ 모형은 변환점 위치 $(20,40)$ 일 때이다.

Figure 3.2 로부터 각 모형의 (a)는 변환점 위치의 상대빈도 히스토그램으로 4 개의 모의실험 자료의 $\mathrm{MAP}$ 모형이 모두 $(20,40)$ 임을 알 수 있고, 또한 $(\mathrm{b})$ 에 의해 MAP 추정치에서 정확히 세 개의 서로 다른 이질적인 영역으로 분리되고 있음을 알 수 있다. (c)와 (d)는 각 변수에서 변환점의 MAP 추정치 인 $(20,40)$ 에 의해 분리된 부분영역들을 보여준다. 따라서 제안된 베이지안 모형에 MHWGS 알고리즘 을 이용하면 정확히 변환점 개수 및 위치를 찾아 낼 수 있음을 알 수 있다.

\section{4. 실증분석}

\section{1. 실증분석 자료}

한우의 맛은 일반적으로 부드러움(Tenderness)과 육즙(Juiciness)으로 맛을 평가한다 (Forrest, 1975). 맛을 결정하는 다양한 변수들 중 근육 내 지방 함량이 한우의 맛을 내는 부드러움과 육즙과 직접 관련되 
Table 3.1. Three five models with the largest log-posterior values in four simulated data set according to the number of change points.

\begin{tabular}{|c|c|c|c|c|c|c|c|}
\hline \multirow[b]{2}{*}{$\mathrm{CP}$} & \multirow[b]{2}{*}{ No } & \multicolumn{3}{|c|}{ (a) } & \multicolumn{3}{|c|}{ (b) } \\
\hline & & $\begin{array}{l}\text { Change } \\
\text { patterns }\end{array}$ & $\begin{array}{c}\text { Log } \\
\text { posterior }\end{array}$ & $\mathrm{BIC}$ & $\begin{array}{l}\text { Change } \\
\text { patterns }\end{array}$ & $\begin{array}{c}\text { Log } \\
\text { posterior }\end{array}$ & $\mathrm{BIC}$ \\
\hline \multirow{5}{*}{1} & 1 & $(20)$ & -113.7180 & 252.0021 & $(20)$ & -117.8768 & 260.3197 \\
\hline & 2 & (20) & -113.7204 & 252.0069 & (20) & -118.1020 & 260.7701 \\
\hline & 3 & (20) & -113.7917 & 252.1495 & (20) & -118.3465 & 261.2591 \\
\hline & 4 & (20) & -113.8655 & 252.2971 & (20) & -118.3475 & 261.2611 \\
\hline & 5 & (20) & -113.9012 & 252.3685 & (20) & -118.4261 & 261.4183 \\
\hline \multirow{5}{*}{2} & 1 & $(20,40)$ & -69.8243 & 176.4977 & $(20,40)$ & -71.0516 & 178.9524 \\
\hline & 2 & $(20,40)$ & -69.9469 & 176.7428 & $(20,40)$ & -71.0703 & 178.9897 \\
\hline & 3 & $(20,40)$ & -69.9916 & 176.8324 & $(20,40)$ & -71.0861 & 179.0213 \\
\hline & 4 & $(20,40)$ & -69.9977 & 176.8444 & $(20,40)$ & -71.2407 & 179.3306 \\
\hline & 5 & $(20,40)$ & -70.4274 & 177.7039 & $(20,40)$ & -71.3068 & 179.4626 \\
\hline \multirow{5}{*}{3} & 1 & $(7,20,40)$ & -79.0371 & 207.2064 & $(20,35,40)$ & -74.9210 & 198.9742 \\
\hline & 2 & $(17,20,40)$ & -79.4618 & 208.0558 & $(20,35,40)$ & -75.1090 & 199.3501 \\
\hline & 3 & $(11,20,40)$ & -79.4962 & 208.1245 & $(20,35,40)$ & -75.1779 & 199.4879 \\
\hline & 4 & $(12,20,40)$ & -79.5388 & 208.2098 & $(20,35,40)$ & -75.2199 & 199.5719 \\
\hline & 5 & $(7,20,40)$ & -79.6206 & 208.3733 & $(20,35,40)$ & -75.2578 & 199.6477 \\
\hline \multirow[b]{2}{*}{$\mathrm{CP}$} & & \multicolumn{3}{|c|}{ (c) } & \multicolumn{3}{|c|}{ (d) } \\
\hline & No & $\begin{array}{l}\text { Change } \\
\text { patterns }\end{array}$ & $\begin{array}{c}\text { Log } \\
\text { posterior }\end{array}$ & $\mathrm{BIC}$ & $\begin{array}{l}\text { Change } \\
\text { patterns }\end{array}$ & $\begin{array}{c}\text { Log } \\
\text { posterior }\end{array}$ & $\mathrm{BIC}$ \\
\hline \multirow{5}{*}{1} & 1 & $(20)$ & -74.9694 & 174.5049 & $(20)$ & -86.4568 & 197.4796 \\
\hline & 2 & (20) & -75.0331 & 174.6323 & (20) & -86.4952 & 197.5565 \\
\hline & 3 & (20) & -75.4836 & 175.5333 & (20) & -86.7231 & 198.0123 \\
\hline & 4 & $(20)$ & -75.4953 & 175.5567 & (20) & -86.8292 & 198.2244 \\
\hline & 5 & $(20)$ & -75.5601 & 175.6862 & $(20)$ & -86.8919 & 198.3498 \\
\hline \multirow{5}{*}{2} & 1 & $(20,40)$ & -31.6007 & 100.0506 & $(20,40)$ & -30.7672 & 98.3835 \\
\hline & 2 & $(20,40)$ & -31.7505 & 100.3501 & $(20,40)$ & -30.7991 & 98.4473 \\
\hline & 3 & $(20,40)$ & -31.7703 & 100.3897 & $(20,40)$ & -30.8469 & 98.5428 \\
\hline & 4 & $(20,40)$ & -31.8245 & 100.4981 & $(20,40)$ & -31.0136 & 98.8762 \\
\hline & 5 & $(20,40)$ & -31.9387 & 100.7265 & $(20,40)$ & -31.1008 & 99.0507 \\
\hline \multirow{5}{*}{3} & 1 & $(16,20,40)$ & -42.0882 & 133.3085 & $(11,20,40)$ & -38.7646 & 126.6614 \\
\hline & 2 & $(6,20,40)$ & -42.1872 & 133.5066 & $(15,20,40)$ & -39.2003 & 127.5327 \\
\hline & 3 & $(6,20,40)$ & -42.2566 & 133.6454 & $(11,20,40)$ & -39.2577 & 127.6475 \\
\hline & 4 & $(5,20,40)$ & -42.3444 & 133.8208 & $(16,20,40)$ & -39.3930 & 127.9181 \\
\hline & 5 & $(16,20,40)$ & -42.4502 & 134.0325 & $(17,20,40)$ & -39.4890 & 128.1101 \\
\hline
\end{tabular}

어 있다고 알려져 있다 (Wheeler 등, 1994). 본 논문은 Cheon과 Kim (2010)에서 사용한 한우자료중 일부를 이용하였으며, 이 자료는 한우의 맛에 영향을 미치는 부드러움과 육즙을 평가하기 위해 각 부위 에서의 지방함량 비율이 측정된 자료이다 (Jennings 등, 1978; Indurain 등, 2009). 한우 맛의 평가 점 수는 0 부터 100 까지이다. Figure 4.1 은 육즙과 부드러움 두 변수가 각각 지방 함량이 높아짐에 따라 얻 어진 그림이다. 본 실증 분석에서는 어느 정도의 지방함량 비율이 한우의 맛의 평가를 변화시키는가를 알기 위해, 본 논문에서 제안한 베이지안 다중 변환점 모형을 이용하여 이변량 자료의 변환점을 찾으려 한다. 

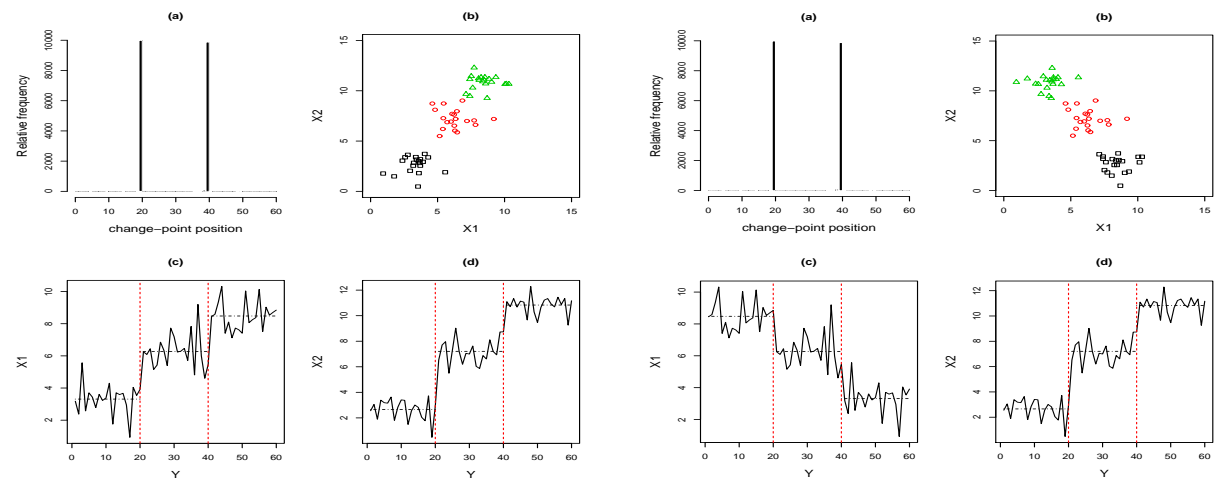

(a)

(b)
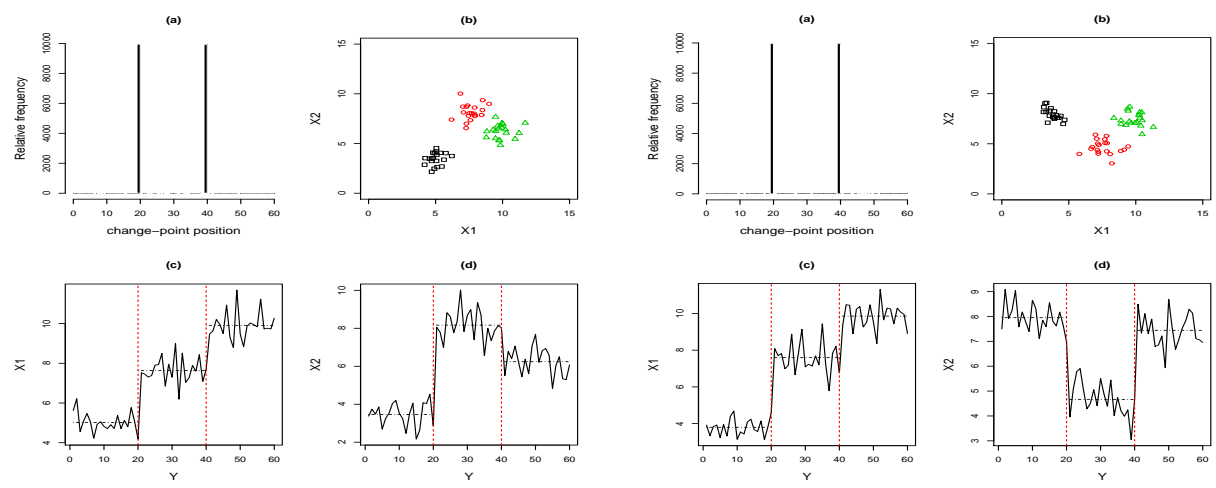

(c)

(d)

Figure 3.2. The simulation results of four multiple change-point examples: (a) The histogram for log posterior probabilities of change-point positions from the posterior samples; (b) A bivariate plot with the maximum logposterior estimate of the change-point positions; (c) A comparison of the MAP estimates of the change-point positions and the true change-point positions in observations of the first variable. The vertical (dotted) lines indicated the change-point positions identified by the MAP model, and the horizontal (dashed) lines indicate the mean value of observations separated by change-point positions of the true model; (d) same with (c) except for the second variable)

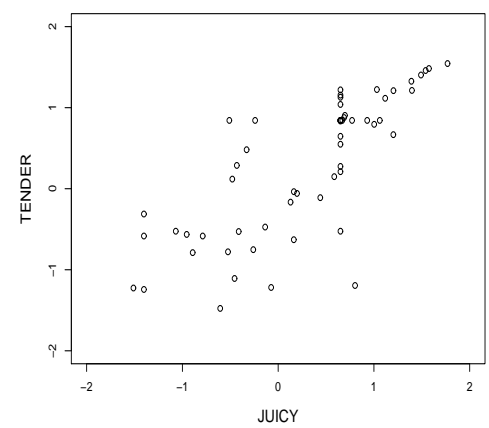

Figure 4.1. A plot of juiciness and tenderness 
Table 4.1. Three ten models with the largest log-posterior values in fat data according to the number of change points.

\begin{tabular}{|c|c|c|c|c|}
\hline $\mathrm{CP}$ & No & Change patterns & Log-posterior & $\mathrm{BIC}$ \\
\hline \multirow{10}{*}{1} & 1 & $(27)$ & -14.7105 & 53.9871 \\
\hline & 2 & $(27)$ & -14.7327 & 54.0315 \\
\hline & 3 & $(27)$ & -14.9964 & 54.5589 \\
\hline & 4 & $(27)$ & -15.0501 & 54.6662 \\
\hline & 5 & $(27)$ & -15.0657 & 54.6974 \\
\hline & 6 & $(22)$ & -15.2512 & 55.0684 \\
\hline & 7 & $(22)$ & -15.2623 & 55.0907 \\
\hline & 8 & $(22)$ & -15.3023 & 55.1707 \\
\hline & 9 & $(27)$ & -15.4431 & 55.4522 \\
\hline & 10 & $(43)$ & -15.4556 & 55.4774 \\
\hline \multirow{10}{*}{2} & 1 & $(43,55)$ & -14.4847 & 65.8185 \\
\hline & 2 & $(43,55)$ & -14.5022 & 65.8534 \\
\hline & 3 & $(43,55)$ & -14.9717 & 66.7925 \\
\hline & 4 & $(43,55)$ & -15.2261 & 67.3012 \\
\hline & 5 & $(43,55)$ & -15.2482 & 67.3454 \\
\hline & 6 & $(43,55)$ & -15.3916 & 67.6323 \\
\hline & 7 & $(43,55)$ & -15.4110 & 67.6711 \\
\hline & 8 & $(42,55)$ & -15.4647 & 67.7785 \\
\hline & 9 & $(42,55)$ & -15.5515 & 67.9521 \\
\hline & 10 & $(43,55)$ & -15.5825 & 68.0142 \\
\hline \multirow{10}{*}{3} & 1 & $(27,43,55)$ & -21.3942 & 91.9204 \\
\hline & 2 & $(27,43,55)$ & -21.4704 & 92.0729 \\
\hline & 3 & $(21,43,55)$ & -21.4708 & 92.0736 \\
\hline & 4 & $(22,43,55)$ & -21.5082 & 92.1485 \\
\hline & 5 & $(27,43,55)$ & -21.5674 & 92.2669 \\
\hline & 6 & $(19,22,55)$ & -21.7648 & 92.6617 \\
\hline & 7 & $(28,43,55)$ & -21.8883 & 92.9088 \\
\hline & 8 & $(24,43,55)$ & -21.9040 & 92.9401 \\
\hline & 9 & $(10,43,55)$ & -21.9071 & 92.9463 \\
\hline & 10 & $(20,43,55)$ & -21.9218 & 92.9758 \\
\hline
\end{tabular}

\section{2. 실증분석 결과}

Table 4.1은 MHWGS 알고리즘을 이용하여 변환점이 1개, 2 개, 3 개인 경우에 각각 10000 번씩 시행하 여 로그사후확률이 가장 큰 10 개 값을 찾아서 그 값에 해당되는 변환점 위치와 로그사후확률및 $\mathrm{BIC}$ 를 구한 결과를 보여주고 있다. Figure $4.2(\mathrm{a})$ 는 변환점 위치의 상대빈도 히스토그램으로 변환점 27 보다 변환점 43 과 55 에서 훨씬 많은 빈도수를 보여 주고 있다. 따라서, 변환점이 2 개이며 변환점 위치 (43, 55 )일 때, 비록 $\mathrm{BIC}$ 값은 비록 제일 작지 않지만 빈도수가 가장 많고 또한 최대 로그사후확률값이 -14.4847 로 가장 크기 때문에 변환점 위치 $(43,55)$ 인 변환점 2 개의 모형이 MAP 모형으로 선택되 었다. 또한, Figure $4.2(\mathrm{~b})$ 로 부터 최적의 모형인 변환점 위치 $(43,55)$ 에서 어느 정도 세 개의 서로 다 른 영역으로 분리하고 있음을 알 수 있다. Figure $4.2(\mathrm{c})$ 와 (d)는 지방함량이 높아짐에 따라 각각 육즙 과 부드러움의 변화를 보여 주고 있으며, 지방함량 변환점의 MAP 추정치인 $(43,55)$ 에 의해 두 변수의 변화를 잘 알 수 있다. 
(a)

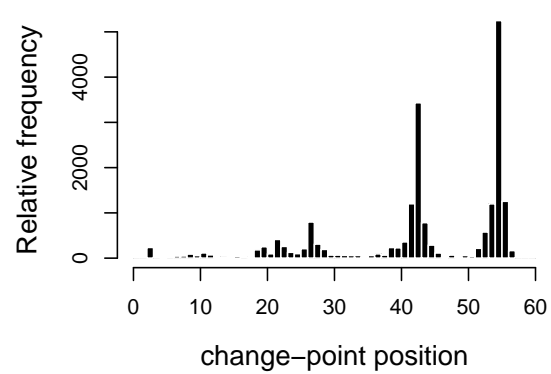

(c)

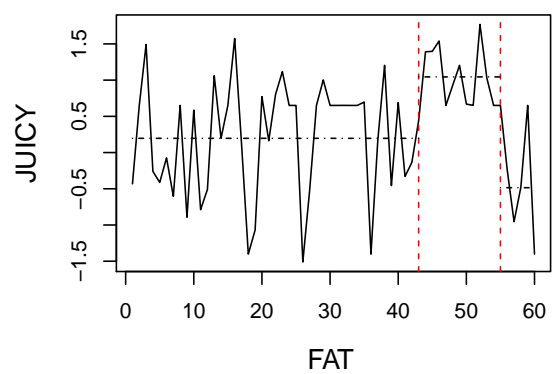

(b)

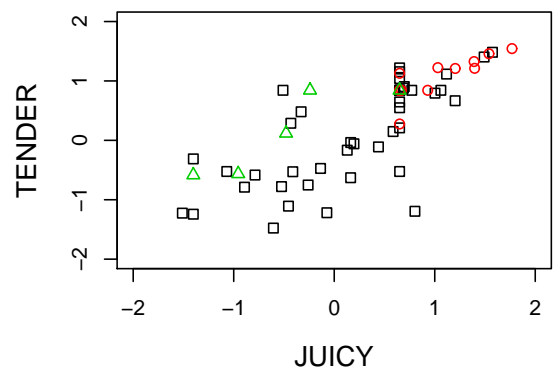

(d)

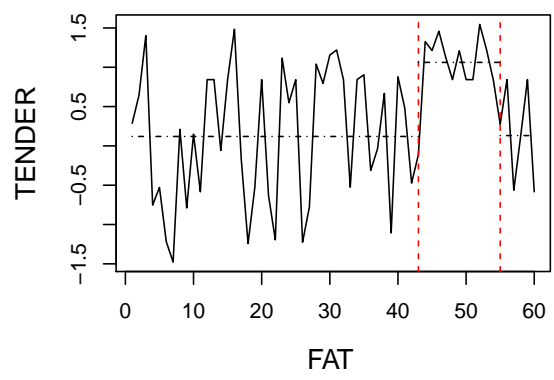

Figure 4.2. The simulation results of multiple change points estimation: (a) The histogram for log posterior probabilities of change points position; (b) A plot of juiciness and tenderness, with the maximum log-posterior estimate of the change-point positions; (c) A plot of the maximum log-posteriori estimated change positions with the change-point positions (the vertical lines) and means (horizontal lines) of juiciness in each subregion; (d) same with (c) except for tenderness)

이 결과는 Cheon과 $\operatorname{Kim}$ (2010)에서 제시한 결과와 비숫한 결과를 보여 준다. Cheon과 $\operatorname{Kim}(2010)$ 은 한우의 지방함량 자료로 272 개 자료를 선택하였다. 하지만 본 논문은 변화가 보다 심한 소량자료에 관 심이 있어서 272 개 자료 중 60 개 자료 (시점, 191-250)만 사용하여 분석하였고, 그 결과 MAP 모형의 변환점 위치 $(43,55)$ 를 찾았고 이 위치는 Cheon과 $\operatorname{Kim}(2010)$ 에서 찾아낸 $(233,245)$ 시점과 정확하 게 일치함을 알 수 있었다. 두 결과의 차이점으로, Cheon과 $\operatorname{Kim}(2010)$ 은 베이지안 다변량 정규분포 를 이용하였고 본 논문에서는 소량자료를 위해 다변량 비중심 $t$ 분포를 사용하였다.

\section{5. 결론}

본 논문에서는 소량자료의 변환점 개수 및 변환점의 위치를 찾고자 할 때 베이지안 다변량 비중심 $t$ 분 포 모형을 제안하였고, Müller $(1991,1993)$ 가 제안한 메트로폴리스-해스팅스를 포함한 깁스 샘플링 알 고리즘을 이용하여 분석을 하였다. 제안된 모형을 모의실험 및 한우의 지방함량자료의 실증 분석에 적 용한 결과, Cheon과 $\mathrm{Kim}$ (2010)의 결과와 비교해 볼때 변환점 개수와 그 위치를 적절하게 잘 찾아주고 있음을 알 수 있었다. 결론적으로 소량자료에 관한 변환점 및 위치를 찾고자 할 때 본 논문의 베이지안 다변량 비중심 $t$ 분포 모형을 제안하며, 또한 결합분포에서 표본을 추출하기 힘들 때 메트로폴리스-해스 팅스를 포함한 깁스 샘플링 알고리즘을 제안하고자 한다. 


\section{References}

Barry, D. and Hartigan, J. A. (1993). A Bayesian analysis for change-point problems, Journal of the American Statistical Association, 88, 309-319.

Carlin, B. P., Gelfand, A. E. and Smith, A. F. M. (1992). Hierarchical Bayesian analysis of change point problem, Applied Statistics, 41, 389-405.

Chen, M. H. and Schmeiser, B. W. (1998). Towards black-box sampling, Journal of Computational and Graphical Statistics, 8, 1-22.

Cheon, S. and Kim, J. (2010). Multiple change-point detection of multivariate mean vectors with the Bayesian approach, Computational Statistics and Data Analysis, 54, 406-415.

Cheon, S. and Yu, W. (2012). Bayesian Multiple change-point for small data, Communications of the Korean Statistical Society, 19, 237-246.

Chernoff, H. and Zacks, S. (1964). Estimating the current mean of a normal distribution which is subject to changes in time, Annals of Mathematical Statistics, 35, 999-1018.

Forrest, R. J. (1975). Effects of castration, sire and hormone treatments on the quality of rib roasts from Holstein-Friesian males, Canadian Journal of Animal Science, 55, 287-290.

Geman, S. and Geman, D. (1984). Stochastic Relaxation, Gibbs Distributions, and Bayesian Restoration of images, IEEE Transactions on Pattern Analysis and Machine Intelligence, 6, 721-741.

Hastings, W. K. (1970). Monte Carlo sampling methods using Markov Chains and their applications, Biometrika, 57, 97-109.

Indurain, G., Carr, T. R., Gonim, M. V., Insausti, K. and Beriain, M. J. (2009). The relationship of carcass measurements to carcass composition and intramuscular fat in Spanish beef, Meat Science, 82, 155-161.

Jennings, T. G., Berry, B. W. and Joseph, A. L. (1978). Influence of fat thickness, marbling and length of aging on beef palatability and shelf-life characteristics, Journal of Animal Science, 46, 658-665.

Kim, J. and Cheon, S. (2010). Bayesian multiple change-point estimation with annealing stochastic approximation Monte Carlo, Computational Statistics, 25, 215-239.

Metropolis, N., Rosenbluth, A. W., Rosenbluth, M. N., Teller, A. H. and Teller, E. (1953). Equations of state calculations by fast computing machines, Journal of Chemical Physics, 21, 1087-1091.

Müller, P. (1991). A Bayesian analysis for change-point problems, A generic approach to posterior integration and Gibbs sampling. Technical Report, Purdue University, West Lafayette IN.

Müller, P. (1993). Alternatives to the Gibbs sampling scheme,Technical, Report, Institute of Statistics and Decision Sciences, Duke University.

Smith, A. F. M. (1975). A Bayesian approach to inference about a change-point in a sequence of random variables, Biometrika, 62, 407-416.

Wheeler, T. L., Cundiff, L. V. and Koch, R. M. (1994). Effect of marbling degree on beef palatability in Bos Taurus and Bos Inbdicus cattle, Journal of Animal Science, 72, 3145-3151. 